\title{
EXISTENCE OF NASH EQUILIBRIA IN TWO-PERSON STOCHASTIC GAMES OF RESOURCE EXTRACTION
}

\author{
PIOTR SZAJOWSKI \\ Institute of Mathematics, Wrockaw University of Technology \\ Wybrzeże Wyspiańskiego 27, 50-370 Wrocław, Poland \\ E-mail:Piotr.Szajowski@pwr.wroc.pl
}

\begin{abstract}
This paper deals with two-person stochastic games of resource extraction under both the discounted and the mean payoff criterion. Under some concavity and additivity assumptions concerning the payoff and the transition probability function a stationary Nash equilibrium is shown to exist. The proof is based on Schauder-Tychonoff's fixed point theorem, applied to a suitable payoff vector space.
\end{abstract}

1. Introduction. The games of resource extraction were introduced by Levhari and Mirman [14]. In such a dynamic game the players extract some amount of common renewable resource at each stage of the game. The amount of available resource in a next stage depends on the amount of the resource left by the players in the previous stage (investment). Extraction of small amount of the resource results in small payoffs of the players. Extraction of great amount of the resource reduces potential payoffs in the future.

In the models of Sundaram [25] or Majumdar and Sundaram [15] the strategies of the players were lower semicontinuous functions of the game state and utilities were the same for all the players. In those models the existence of Nash equilibria in pure stationary strategies was proved.

A similar model was considered by Amir [1]. He used the Topkis Theorem (see [27])— about monotonicity of a maximand of a submodular function on a lattice-and the Schauder fixed point theorem (see [23]).

The models of resource extraction games were also considered by Dutta and Sundaram [9], Cave [6], Fisher and Mirman [11], Mendelsohn and Sobel [16], Sobel [24] and Więcek [28].

2000 Mathematics Subject Classification: Primary 91A15.

This paper is a part of the dissertation of the author. The author wishes to thank his supervisor, Prof. Andrzej Nowak, for inspiration and much help.

The paper is in final form and no version of it will be published elsewhere. 
This paper also deals with such models. Unlike in the papers of Amir [1,2], the state space of the game does not have to be unbounded from above. The main assumptions deal with the structure of transition probabilities (they have to be additive) and utility functions of the players. The existence of a stationary pure Nash equilibria in both cases of discounted and - under some additional assumptions - mean payoffs are proved.

Some models with similar transition probability structure were also considered by Nowak and Szajowski [22] and Szajowski [26]. In those papers it is assumed that there exists an absorbing state of total resource extraction (of resource level zero). This additional assumption allows for a construction of sequences converging to Nash equilibria. However, in the model from this paper it was not the case. Similar approach can be found in the paper of Nowak [20] and also in the one of Balbus and Nowak [3], in which the symmetric games were considered.

A survey on the Nash and correlated equilibria in stochastic games with infinite state space can be found in the paper of Nowak [21].

This paper is organized in the following way: in the first section we define the model, in the second one we formulate and prove the main results concerning the discounted game model, and in the third one, the results concerning the model with mean payoffs. In the last section and in the appendix the results needed for the proofs in the previous sections are discussed.

2. The model of the game - definitions, notation and assumptions. In this paper we deal with a model of a stochastic game of resource extraction:

DEFinition 2.1. The 2-person stochastic game of resource extraction is defined as a 4-tuple $(S, D, \bar{u}, p)$ of the following objects:

(i) $S$ is an interval in $(0, \infty)$ open from the left (called later the set of all possible resource stocks or the state space),

(ii) $D:=\left\{(s, \bar{x}): s \in S, \bar{x}=\left(x_{1}, x_{2}\right), x_{i} \in A_{i}(s)\right.$ for $\left.i=1,2\right\}$, where $A_{i}(s):=$ $\left[0, a_{i}(s)\right]$ represents the set of admissible decisions of player $i$ in state $s \in S$. The functions $a_{i}: S \rightarrow R_{+}:=[0,+\infty)$ are Borel and satisfy

$$
a_{1}(s)+a_{2}(s)<s-\inf S \text { for each } s \in S .
$$

(iii) $\bar{u}=\left(u_{1}, u_{2}\right)$, where $u_{i}: R_{+} \rightarrow R_{+}$(for $\left.i=1,2\right)$ is a continuous utility function of player $i$,

(iv) $p$ is a transition probability from the set $D$ into $S$.

The game takes place in discrete time. If in some moment the state of the game is $s$, then player $i$ chooses $x_{i} \in A_{i}(s) \subseteq R_{+}$and obtains the payoff $u_{i}\left(x_{i}\right)$ and the state of the game changes according to the transition probability distribution $p(\cdot \mid s, \bar{x})$, where $\bar{x}=\left(x_{1}, x_{2}\right)$.

$x_{i}$ may be interpreted as an amount of resource consumed by the player $i$ in that moment. It must be nonnegative and is bounded from above by the quantity $a_{i}(s)$, which represents the maximal admissible level of consumption of player $i$ in state $s$. If the game is in a state $s$, it reflects the situation that the amount $s$ of the resource is present in the environment. The common level of players' consumption should not lead to overcon- 
sumption, that is, the amount of the resource after players' consumption must be greater than some minimal level.

Definition 2.2. Let $H^{1}=S$ and for $n \geq 2$

$$
H^{n}=D_{1} \times \cdots \times D_{n-1} \times S,
$$

where $D_{k}:=D, k=1, \cdots, n-1 . H^{n}$ is called the space of histories of the game up to the $n$-th state.

Let $H^{\infty}=D \times D \times \cdots$ This is called the space of all histories of the infinite horizon game.

REMARK 2.1. (a) The spaces $H^{n}$ and $H^{\infty}$ are endowed with the product $\sigma$-algebras.

(b) This paper deals with the models of investment/consumption in which randomized strategies do not have natural interpretation. Therefore we shall restrict attention to non-randomized strategies.

(c) The players have full information about the entire history of the game at any stage.

Definition 2.3. A strategy of player $i$ is defined as a sequence

$$
\pi_{i}=\left(\pi_{i, 1}, \pi_{i, 2}, \cdots\right),
$$

where $\pi_{i, n}$ is a Borel mapping from $H^{n}$ into $[0,+\infty)$ such that for every

$$
h^{n}=\left(s_{1}, x_{1,1}, x_{2,1}, s_{2}, \cdots, s_{n}\right) \in H^{n},
$$

we have

$$
\pi_{i, n}\left(h^{n}\right) \in A_{i}\left(s_{n}\right) .
$$

The set of strategies of player $i$ will be denoted by $\Pi_{i}$.

Definition 2.4. A strategy $\pi_{i}=\left(\pi_{i, 1}, \pi_{i, 2}, \cdots\right)$ of player $i$ such that $\pi_{i, n}$ depends on $n$ and the state $s_{n}$ (in the current moment $n$ ) only will be called Markovian.

Definition 2.5. A Markovian strategy of player $i$ of the form $\pi_{i}=(f, f, \cdots)$, where $f$ associates with any $s \in S$ a point $f(s) \in A_{i}(s)$ will be called stationary.

The set of stationary strategies of player $i$ will be denoted by $F_{i}$.

For every strategy profile $\bar{\pi}=\left(\pi_{i}\right)_{i=1}^{2}$ of both players and any initial state of the game $s_{1}=s \in S$ the stochastic process $\left\{\left(s_{n}, \bar{x}_{n}\right)\right\}$ (where $\left.\bar{x}_{n}=\left(x_{1, n}, x_{2, n}\right)\right)$ is defined on $H^{\infty}$ in the canonical way (see Chapter 7 in [4]), where the random variables $s_{n}$ and $\bar{x}_{n}$ are the state of the game and the profile of players' strategies on $n$-th step of the game, respectively.

REMARK 2.2. Existence of the stochastic process $\left\{\left(s_{n}, \bar{x}_{n}\right)\right\}$ and the unique probability measure on $H^{\infty}$ generated by the initial state of the game $s$ and the transition probabilities follow from the Ionescu-Tulcea Theorem (see Proposition V.1.1 in Neveu [19]).

Let us denote the above mentioned probability measure by $P_{s}^{\bar{\pi}}$ and the expected value operator associated with this measure by $E_{s}^{\bar{\pi}}$. 
Definition 2.6. Put $\Pi=\Pi_{1} \times \Pi_{2}$, choose $\bar{\pi} \in \Pi$. Let $\beta$ be a discount factor. Assuming that $\beta \in(0,1)$, define the $\beta$-discounted expected payoff of player $i$ in the infinite horizon game as

$$
\gamma_{i}(\bar{\pi})(s):=E_{s}^{\bar{\pi}}\left(\sum_{n=1}^{\infty} \beta^{n-1} u_{i}\left(x_{i, n}\right)\right),
$$

where $E_{s}^{\bar{\pi}}$ is the expected value operator with respect to the probability measure $P_{s}^{\bar{\pi}}$ and $x_{i, n}$ is the $i$-th coordinate of the vector $\bar{x}_{n}$.

DeFinition 2.7. The expected mean payoff of player $i$ is defined as

$$
\phi_{i}(\bar{\pi})(s):=\liminf _{m \rightarrow \infty} \frac{E_{s}^{\bar{\pi}}\left(\sum_{n=1}^{m} u_{i}\left(x_{i, n}\right)\right)}{m} .
$$

Definition 2.8. A strategy profile $\bar{\pi}^{*} \in \Pi$ is called a Nash equilibrium in the discounted infinite horizon stochastic game iff

$$
\begin{aligned}
& \gamma_{1}\left(\bar{\pi}^{*}\right)(s) \geq \gamma_{1}\left(\pi_{1}, \pi_{2}^{*}\right)(s), \\
& \gamma_{2}\left(\bar{\pi}^{*}\right)(s) \geq \gamma_{2}\left(\pi_{1}^{*}, \pi_{2}\right)(s)
\end{aligned}
$$

for all $\pi_{i} \in \Pi_{i}, s \in S$.

A Nash equilibrium in the infinite horizon stochastic game with the expected mean payoff functions is defined analogously.

In this paper we make the following additional assumptions:

Assumption 2.1. For every $\left(s, x_{1}, x_{2}\right) \in D$ the transition probability $p\left(\cdot \mid s, x_{1}, x_{2}\right)$ has the form

$$
p\left(\cdot \mid s, x_{1}, x_{2}\right)=l\left(s-x_{1}-x_{2}\right) H_{1}(\cdot \mid s)+\left(1-l\left(s-x_{1}-x_{2}\right)\right) H_{2}(\cdot \mid s),
$$

where

(i) $l: S \rightarrow[0,1]$ is increasing, concave and twice differentiable,

(ii) $H_{1}(\cdot \mid s), H_{2}(\cdot \mid s)$ are transition probabilities from $S$ into $S$ such that there is a probability measure $\mu$ on the set $S$, for which

$$
H_{i}(\cdot \mid s) \ll \mu(\cdot), \quad i=1,2 .
$$

The term $l\left(s-x_{1}-x_{2}\right)$ indicates (indirectly) how much of the resource is left after players' consumption in a previous stage. So, the influence of the transition probability $H_{1}$ on the probabiliy $p$ increases with the common players' investment and the influence of $\mathrm{H}_{2}$ increases with the consumption.

Additionally we assume that the utility functions satisfy:

Assumption 2.2. $u_{i}$ are increasing, nonnegative, bounded, twice differentiable (at zerofrom the right-hand side) and $u_{i}^{\prime \prime}(x)<0$ for each $x \in(0,+\infty)$.

By $b>0$ we shall denote a common upper bound of the functions $u_{i}, i=1,2$.

\section{Nash equilibria in discounted stochastic games}

THEOREM 3.1. Suppose that Assumptions 2.1 and 2.2 hold in the considered model of the stochastic game with the $\beta$-discounted expected payoff. Then there exists a stationary Nash equilibrium $\left(f_{1}^{*}, f_{2}^{*}\right)$ in this game. 
For the proof two additional definitions and a lemma are needed. First, consider an auxiliary game:

DEFINITION 3.1. Let $\Gamma\left(v_{1}, v_{2}, s\right)$ be a one-shot game, where the payoff function of player $i$ is:

$$
r_{v_{i}}^{i}\left(s, x_{1}, x_{2}\right):=(1-\beta) u_{i}\left(x_{i}\right)+\beta \int_{S} v_{i}\left(s^{\prime}\right) p\left(d s^{\prime} \mid s, x_{1}, x_{2}\right)
$$

where

$$
\begin{gathered}
\left(v_{1}, v_{2}\right) \in B:=B_{1} \times B_{2}, \\
B_{i}:=\left\{v: S \rightarrow R_{+}:\|v\|_{\infty} \leq b\right\}
\end{gathered}
$$

and

$$
\|v\|_{\infty}:=\operatorname{esssup}_{s \in S}|v(s)| .
$$

It is obvious that $B_{i} \subseteq L^{\infty}(\mu)$. Endow $L^{\infty}(\mu)$ with the weak*-topology. It is well known that a sequence $\left\{f_{n}\right\} \subset L^{\infty}(\mu)$ converges to $f \in L^{\infty}(\mu)$ in weak*-topology iff for all $g \in L^{1}(\mu)$

$$
\lim _{n \rightarrow \infty} \int_{S} f_{n}(s) g(s) \mu(d s)=\int_{S} f(s) g(s) \mu(d s) .
$$

Remark 3.1. It is obvious that $B$ is convex. From the Banach-Alaoglu Theorem (see [8]), it follows that $B_{i}$ is compact in the relative weak*-topology. Hence $B=B_{1} \times B_{2}$ is also compact.

Definition 3.2. Let $\left(v_{1}, v_{2}\right) \in B$. Put

$$
\begin{gathered}
w_{i}(s):=r_{v_{i}}^{i}\left(s, \tilde{x}_{1}(s), \tilde{x}_{2}(s)\right), \\
\tilde{x}_{1}(s):=\underset{x_{1} \in\left[0, a_{1}(s)\right]}{\operatorname{argmax}} r_{v_{1}}^{1}\left(s, x_{1}, \tilde{x}_{2}(s)\right), \\
\tilde{x}_{2}(s):=\underset{x_{2} \in\left[0, a_{2}(s)\right]}{\operatorname{argmax}} r_{v_{2}}^{2}\left(s, \tilde{x}_{1}(s), x_{2}\right) .
\end{gathered}
$$

Define

$$
W\left(v_{1}, v_{2}\right):=\left(w_{1}, w_{2}\right) .
$$

The above definition is correct, because if $s \in S$ is fixed, then the game $\Gamma\left(v_{1}, v_{2}, s\right)$ fulfils the assumptions of Lemma 5.3. Thus there exists a unique Nash equilibrium $\left(\tilde{x}_{1}(s), \tilde{x}_{2}(s)\right)$ in $\Gamma\left(v_{1}, v_{2}, s\right)$.

Lemma 3.1. The mapping $W$ is continuous when $B$ is endowed with the product weak*topology.

Proof. Consider a sequence $\left\{\left(v_{1}^{n}, v_{2}^{n}\right)\right\} \subset B$ converging to some $\left(v_{1}, v_{2}\right) \in B$. We will show that the sequence $\left\{W\left(v_{1}^{n}, v_{2}^{n}\right)\right\}$ converges to $W\left(v_{1}, v_{2}\right) \in B$.

Denote:

$$
\begin{aligned}
& \tilde{x}_{1}^{n}(s):=\underset{x_{1} \in\left[0, a_{1}(s)\right]}{\operatorname{argmax}} r_{v_{1}^{n}}^{1}\left(s, x_{1}, \tilde{x}_{2}^{n}(s)\right), \\
& \tilde{x}_{2}^{n}(s):=\underset{x_{2} \in\left[0, a_{2}(s)\right]}{\operatorname{argmax}} r_{v_{2}^{n}}^{2}\left(s, \tilde{x}_{1}^{n}(s), x_{2}\right),
\end{aligned}
$$




$$
\begin{aligned}
& \tilde{x}_{1}(s):=\underset{x_{1} \in\left[0, a_{1}(s)\right]}{\operatorname{argmax}} r_{v_{1}}^{1}\left(s, x_{1}, \tilde{x}_{2}(s)\right), \\
& \tilde{x}_{2}(s):=\underset{x_{2} \in\left[0, a_{2}(s)\right]}{\operatorname{argmax}} r_{v_{2}}^{2}\left(s, \tilde{x}_{1}(s), x_{2}\right) .
\end{aligned}
$$

$\left(\tilde{x}_{1}^{n}(s), \tilde{x}_{2}^{n}(s)\right)$ is a Nash equilibrium in the game $\Gamma\left(v_{1}^{n}, v_{2}^{n}, s\right)$, and $\left(\tilde{x}_{1}(s), \tilde{x}_{2}(s)\right)$ in the game $\Gamma\left(v_{1}, v_{2}, s\right)$. Lemma 5.3 implies that these Nash equilibria are unique.

Note that for fixed $s \in S$ the sequence $r_{v_{i}^{n}}^{i}\left(s, x_{1}, x_{2}\right)$ converges to $r_{v_{i}}^{i}\left(s, x_{1}, x_{2}\right)$ (as $n \rightarrow \infty)$ uniformly in $x_{1}$ and $x_{2}$ :

$$
\begin{aligned}
\left|r_{v_{1}^{n}}^{1}\left(s, x_{1}, x_{2}\right)-r_{v_{1}}^{1}\left(s, x_{1}, x_{2}\right)\right|= & \mid(1-\beta) u_{1}\left(x_{1}\right)+\beta\left[\int_{S} v_{1}^{n}\left(s^{\prime}\right) l\left(s-x_{1}-x_{2}\right) H_{1}\left(d s^{\prime} \mid s\right)\right. \\
& \left.+\int_{S} v_{1}^{n}\left(s^{\prime}\right)\left(1-l\left(s-x_{1}-x_{2}\right)\right) H_{2}\left(d s^{\prime} \mid s\right)\right] \\
& -(1-\beta) u_{1}\left(x_{1}\right)-\beta\left[\int_{S} v_{1}\left(s^{\prime}\right) l\left(s-x_{1}-x_{2}\right) H_{1}\left(d s^{\prime} \mid s\right)\right. \\
& \left.+\int_{S} v_{1}\left(s^{\prime}\right)\left(1-l\left(s-x_{1}-x_{2}\right)\right) H_{2}\left(d s^{\prime} \mid s\right)\right] \mid \\
= & \beta \mid l\left(s-x_{1}-x_{2}\right) \int_{S}\left[v_{1}^{n}\left(s^{\prime}\right)-v_{1}\left(s^{\prime}\right)\right] H_{1}\left(d s^{\prime} \mid s\right) \\
& +\left(1-l\left(s-x_{1}-x_{2}\right)\right) \int_{S}\left[v_{1}^{n}\left(s^{\prime}\right)-v_{1}\left(s^{\prime}\right)\right] H_{2}\left(d s^{\prime} \mid s\right) \mid .
\end{aligned}
$$

By the Radon-Nikodym Theorem, for any fixed state $s \in S$, the probability measure $H_{j}(\cdot \mid s)$ has a density function, say $g_{j}$. Therefore (1) can be rewritten in the following way:

$$
\begin{aligned}
\left|r_{v_{1}^{n}}^{1}\left(s, x_{1}, x_{2}\right)-r_{v_{1}}^{1}\left(s, x_{1}, x_{2}\right)\right| & =\beta \mid l\left(s-x_{1}-x_{2}\right) \int_{S}\left[v_{1}^{n}\left(s^{\prime}\right)-v_{1}\left(s^{\prime}\right)\right] g_{1}\left(s^{\prime}\right) \mu\left(d s^{\prime}\right) \\
& +\left(1-l\left(s-x_{1}-x_{2}\right)\right) \int_{S}\left[v_{1}^{n}\left(s^{\prime}\right)-v_{1}\left(s^{\prime}\right)\right] g_{2}\left(s^{\prime}\right) \mu\left(d s^{\prime}\right) \mid .
\end{aligned}
$$

Since $l\left(s-x_{1}-x_{2}\right) \in[0,1]$ and the integrals in (2) converge to zero as $n \rightarrow \infty$ (by the convergence of the sequence $\left\{\left(v_{1}^{n}\right)\right\}$ to $v_{1}$ in the weak-topology) we can conclude that $r_{v_{1}^{n}}^{1}\left(s, x_{1}, x_{2}\right)$ converges uniformly to $r_{v_{1}}^{1}\left(s, x_{1}, x_{2}\right)$. The case of the sequence $\left\{r_{v_{2}^{n}}^{2}\left(s, x_{1}, x_{2}\right)\right\}$ follows along the same lines.

Fix $s \in S$. For any $n \geq 1, x_{1} \in\left[0, a_{1}(s)\right]$ and $x_{2} \in\left[0, a_{2}(s)\right]$ we have

$$
\begin{aligned}
& r_{v_{1}^{n}}^{1}\left(s, x_{1}, \tilde{x}_{2}^{n}(s)\right) \leq r_{v_{1}^{n}}^{1}\left(s, \tilde{x}_{1}^{n}(s), \tilde{x}_{2}^{n}(s)\right), \\
& r_{v_{2}^{n}}^{2}\left(s, \tilde{x}_{1}^{n}(s), x_{2}\right) \leq r_{v_{2}^{n}}^{2}\left(s, \tilde{x}_{1}^{n}(s), \tilde{x}_{2}^{n}(s)\right) .
\end{aligned}
$$

Let $\left(x_{1}^{0}(s), x_{2}^{0}(s)\right)$ be any accumulation point of the sequence $\left\{\left(\tilde{x}_{1}^{n}(s), \tilde{x}_{2}^{n}(s)\right)\right\}$. Then there exists a subsequence $\left\{\left(\tilde{x}_{1}^{k_{n}}(s), \tilde{x}_{2}^{k_{n}}(s)\right)\right\}$ such that

$$
\lim _{n \rightarrow \infty} \tilde{x}_{1}^{k_{n}}(s)=x_{1}^{0}(s), \quad \lim _{n \rightarrow \infty} \tilde{x}_{2}^{k_{n}}(s)=x_{2}^{0}(s) .
$$

Obviously, with $\tilde{x}_{1}^{k_{n}}(s)$ and $\tilde{x}_{2}^{k_{n}}(s)$ the inequalities (3) and (4) hold. From the unifom convergence of the sequences $\left\{r_{v_{1}^{n}}^{1}\left(s, x_{1}, x_{2}\right)\right\}$ and $\left\{r_{v_{2}^{n}}^{2}\left(s, x_{1}, x_{2}\right)\right\}$ we can conclude:

$$
r_{v_{1}}^{1}\left(s, x_{1}, x_{2}^{0}(s)\right) \leq r_{v_{1}}^{1}\left(s, x_{1}^{0}(s), x_{2}^{0}(s)\right), \quad r_{v_{2}}^{2}\left(s, x_{1}^{0}(s), x_{2}\right) \leq r_{v_{2}}^{2}\left(s, x_{1}^{0}(s), x_{2}^{0}(s)\right),
$$


for any $x_{1} \in\left[0, a_{1}(s)\right]$ and $x_{2} \in\left[0, a_{2}(s)\right]$. It means that $\left(x_{1}^{0}(s), x_{2}^{0}(s)\right)$ is a Nash equilibrium in the game $\Gamma\left(v_{1}, v_{2}, s\right)$. Lemma 5.3 implies that the equilibrium is unique, so we obtain

$$
\lim _{n \rightarrow \infty}\left(\tilde{x}_{1}^{n}(s), \tilde{x}_{2}^{n}(s)\right)=\left(\tilde{x}_{1}(s), \tilde{x}_{2}(s)\right)
$$

and

$$
w_{i}(s)=r_{v_{i}}^{i}\left(s, \tilde{x}_{1}(s), \tilde{x}_{2}(s)\right)=\lim _{n \rightarrow \infty} r_{v_{i}^{n}}^{i}\left(s, \tilde{x}_{1}^{n}(s), \tilde{x}_{2}^{n}(s)\right)=\lim _{n \rightarrow \infty} w_{i}^{n}(s) .
$$

By the Lebesgue dominated convergence theorem $w_{i}^{n} \rightarrow w$ in the weak*-topology on $B_{i}$. Thus the result follows.

Proof of Theorem 3.1. The above lemmas and the Schauder-Tychonoff Fixed Point Theorem (see [23]) imply the existence of $v_{i} \in B_{i}$ such that

$$
W\left(v_{1}, v_{2}\right)=\left(v_{1}, v_{2}\right) \quad \mu \text {-a.e. }
$$

Let $\left(f_{1}^{*}(s), f_{2}^{*}(s)\right)$ be the unique Nash equilibrium in the game $\Gamma\left(v_{1}, v_{2}, s\right), s \in S$. Then from (5), it follows that

$$
\begin{aligned}
& v_{1}(s)=\max _{x_{1} \in\left[0, a_{1}(s)\right]}\left[(1-\beta) u_{1}\left(x_{1}\right)+\beta \int_{S} v_{1}\left(s^{\prime}\right) p\left(d s^{\prime} \mid s, x_{1}, f_{2}^{*}(s)\right)\right], \\
& v_{2}(s)=\max _{x_{2} \in\left[0, a_{2}(s)\right]}\left[(1-\beta) u_{2}\left(x_{2}\right)+\beta \int_{S} v_{2}\left(s^{\prime}\right) p\left(d s^{\prime} \mid s, f_{1}^{*}(s), x_{2}\right)\right]
\end{aligned}
$$

for all $s \in S \backslash E$ with $\mu(E)=0$.

Let $\left(v_{1}^{*}, v_{2}^{*}\right)$ be the pair of the Nash equilibrium payoffs in the game $\Gamma\left(v_{1}, v_{2}, s\right)$ for every $s \in S$. We have $v_{i}^{*}(s)=v_{i}(s)$ for each $s \in S \backslash E$, so the pair $\left(f_{1}^{*}(s), f_{2}^{*}(S)\right)$ is also the Nash equilibrium point in the game $\Gamma\left(v_{1}^{*}, v_{2}^{*}, s\right)$ for $s \in S \backslash E$. Observe that since $p\left(\cdot \mid s, x_{1}, x_{2}\right) \ll \mu(\cdot)$ for every $s \in S, x_{i} \in A_{i}(s), i=1,2$. We have

$$
\begin{aligned}
v_{1}^{*}(s) & =\max _{x_{1} \in\left[0, a_{1}(s)\right]}\left[(1-\beta) u_{1}\left(x_{1}\right)+\beta \int_{S} v_{1}\left(s^{\prime}\right) p\left(d s^{\prime} \mid s, x_{1}, f_{2}^{*}(s)\right)\right] \\
& =\max _{x_{1} \in\left[0, a_{1}(s)\right]}\left[(1-\beta) u_{1}\left(x_{1}\right)+\beta \int_{S} v_{1}^{*}\left(s^{\prime}\right) p\left(d s^{\prime} \mid s, x_{1}, f_{2}^{*}(s)\right)\right], \\
v_{2}^{*}(s) & =\max _{x_{2} \in\left[0, a_{2}(s)\right]}\left[(1-\beta) u_{2}\left(x_{2}\right)+\beta \int_{S} v_{2}\left(s^{\prime}\right) p\left(d s^{\prime} \mid s, f_{1}^{*}(s), x_{2}\right)\right] \\
& =\max _{x_{2} \in\left[0, a_{2}(s)\right]}\left[(1-\beta) u_{2}\left(x_{2}\right)+\beta \int_{S} v_{2}^{*}\left(s^{\prime}\right) p\left(d s^{\prime} \mid s, f_{1}^{*}(s), x_{2}\right)\right]
\end{aligned}
$$

for each $s \in S$.

Let $w_{i}^{*}(s)=\frac{v_{i}^{*}(s)}{1-\beta}$. The above equations may be rewritten in the form

$$
\begin{aligned}
& w_{1}^{*}(s)=\max _{x_{1} \in\left[0, a_{1}(s)\right]}\left[u_{1}\left(x_{1}\right)+\beta \int_{S} w_{1}^{*}\left(s^{\prime}\right) p\left(d s^{\prime} \mid s, x_{1}, f_{2}^{*}(s)\right)\right], \\
& w_{2}^{*}(s)=\max _{x_{2} \in\left[0, a_{2}(s)\right]}\left[u_{2}\left(x_{2}\right)+\beta \int_{S} w_{2}^{*}\left(s^{\prime}\right) p\left(d s^{\prime} \mid s, f_{1}^{*}(s), x_{2}\right)\right],
\end{aligned}
$$

for every $s \in S$. 
From Blackwell's work on discounted dynamic programming [5] and the above equations $((8)$ and $(9))$, it follows that

$$
w_{1}^{*}(s)=\gamma_{1}\left(f_{1}^{*}, f_{2}^{*}\right)(s)=\max _{\pi_{1} \in \Pi_{1}} \gamma_{1}\left(\pi_{1}, f_{2}^{*}\right)(s)
$$

and

$$
w_{2}^{*}(s)=\gamma_{2}\left(f_{1}^{*}, f_{2}^{*}\right)(s)=\max _{\pi_{2} \in \Pi_{2}} \gamma_{2}\left(f_{1}^{*}, \pi_{2}\right)(s)
$$

for each $s \in S$. So the pair $\left(f_{1}^{*}, f_{2}^{*}\right)$ is also a Nash equilibrium in the stochastic game with the $\beta$-discounted expected payoff.

4. Nash equilibrium in stochastic games with expected mean payoffs. Let us make an additional assumption on our game model:

Assumption 4.1. (i) The probabilities $H_{i}(\cdot \mid s)$ do not depend on $s$ (for simplicity of notation we shall denote them by $\left.H_{i}(\cdot)\right)$.

(ii) There exist a probability measure $\nu$ on $S$ and a constant $\delta \in(0,1)$ such that for any measurable set $A \subseteq S$ we have

$$
H_{i}(A) \geq \delta \nu(A)
$$

for any $i \in\{1,2\}$.

REMARK 4.1. From the above assumption we conclude immediately that $p\left(A \mid s, x_{1}, x_{2}\right) \geq$ $\delta \nu(A)$ and

$$
H_{i} \ll \mu:=\frac{H_{1}+H_{2}}{2} .
$$

THEOREM 4.1. If Assumptions 2.1, 2.2 and 4.1 hold in our model of the infinite horizon game with the expected mean payoffs of the players, then a Nash equilibrium exists.

Proof. Note that for the discounted game in which the transition probabilities are of the form

$$
\tilde{p}\left(\cdot \mid s, x_{1}, x_{2}\right)=\frac{p\left(\cdot \mid s, x_{1}, x_{2}\right)-\delta \nu(\cdot)}{1-\delta}
$$

and the discount factor is equal $\beta=1-\delta$, both Assumptions 2.1 and 2.2 are satisfied.

Theorem 3.1 implies that in such a game there exists a stationary Nash equilibrium $\left(f_{1}^{*}, f_{2}^{*}\right)$ and the Bellman optimality equations are satisfied:

$$
\begin{aligned}
& v_{1}(s)=\max _{x_{1} \in\left[0, a_{1}(s)\right]}\left[u_{1}\left(x_{1}\right)+(1-\delta) \int_{S} v_{1}\left(s^{\prime}\right) \tilde{p}\left(d s^{\prime} \mid s, x_{1}, f_{2}^{*}(s)\right)\right], \\
& v_{2}(s)=\max _{x_{2} \in\left[0, a_{2}(s)\right]}\left[u_{2}\left(x_{2}\right)+(1-\delta) \int_{S} v_{2}\left(s^{\prime}\right) \tilde{p}\left(d s^{\prime} \mid s, f_{1}^{*}(s), x_{2}\right)\right] .
\end{aligned}
$$

Denote:

$$
d_{i}:=\delta \int_{S} v_{i}\left(s^{\prime}\right) \nu\left(d s^{\prime}\right)
$$


Equations (10) and (11) can be rewritten in the following form:

$$
\begin{aligned}
& v_{1}(s)=\max _{x_{1} \in\left[0, a_{1}(s)\right]}\left[u_{1}\left(x_{1}\right)+\int_{S} v_{1}\left(s^{\prime}\right) p\left(d s^{\prime} \mid s, x_{1}, f_{2}^{*}(s)\right)-d_{1}\right], \\
& v_{2}(s)=\max _{x_{2} \in\left[0, a_{2}(s)\right]}\left[u_{2}\left(x_{2}\right)+\int_{S} v_{2}\left(s^{\prime}\right) p\left(d s^{\prime} \mid s, f_{1}^{*}(s), x_{2}\right)-d_{2}\right] .
\end{aligned}
$$

These are the optimality equations for the game with the expected mean payoffs (see [13]). Thus $\left(f_{1}^{*}, f_{2}^{*}\right)$ is a stationary Nash equilibrium in the infinite game with the expected mean payoffs and the Nash equilibrium payoff of player $i$ in this game is $\phi_{i}\left(f_{1}^{*}, f_{2}^{*}\right)(s)=d_{i}$ (it does not depend on $s$ ).

REMARK 4.2. (a) The transformation to the discounted game used in the proof was introduced in dynamic programming by Dynkin and Yushkevich in their book [10].

(b) Assumption 4.1 implies that the Markov process generated by the strategies in the Nash equilibrium $\left(f_{1}^{*}, f_{2}^{*}\right)$ is geometrically ergodic (see [13]) and has a stationary distribution. The existence of a stationary distribution is an important question in studying various economic models, see $[2,7]$.

5. The uniqueness of the Nash equilibria in a one-shot game. In this section we shall consider the problem of uniqueness of the Nash equilibrium in an auxiliary game.

In the lemmas below we shall consider functions

$$
w_{i}: X_{1} \times X_{2} \rightarrow R
$$

for $i=1,2$, where $X_{1}, X_{2}$ are compact intervals. The function $w_{i}$ is strictly concave in the $i$-th variable and its second partial derivatives are continuous.

REMARK 5.1. From the well known result of Nash (see [18]) the two-person one-shot game with the payoff functions $w_{i}$ and the actions sets $X_{i}$ has a Nash equilibrium.

Assumption 5.1. Assume that for any $\bar{x}=\left(x_{1}, x_{2}\right) \in X_{1} \times X_{2}$ and $i=1,2$ it holds

$$
\left|\frac{\partial^{2} w_{i}}{\partial x_{i}^{2}}(\bar{x})\right|>\left|\frac{\partial^{2} w_{i}}{\partial x_{i} \partial x_{3-i}}(\bar{x})\right| .
$$

For any $\bar{x}=\left(x_{1}, x_{2}\right) \in X_{1} \times X_{2}$ define the norm

$$
\|\bar{x}\|_{\infty}=\max _{i \in\{1,2\}}\left|x_{i}\right| .
$$

The first lemma in this section is a result of Moré (see [17]). The proof of this lemma, based on the Rolle Theorem can be found in the paper of Gabay and Moulin [12].

Lemma 5.1 (Moré). If Assumption 5.1 holds and $\bar{x}, \bar{y} \in X_{1} \times X_{2}$ are different, then

$$
\frac{\partial w_{i}}{\partial x_{i}}(\bar{x})=\frac{\partial w_{i}}{\partial x_{i}}(\bar{y})
$$

implies that

$$
\left|x_{i}-y_{i}\right|<\|\bar{x}-\bar{y}\|_{\infty} .
$$

The following lemma is a slight modification of Theorem 4.1 from [12]. 
Lemma 5.2. Let Assumption 5.1 be fulfilled and $w_{i}$ be strictly concave in $x_{i}$. Then in the two-person game in which $w_{i}\left(x_{1}, x_{2}\right)$ are the payoffs of the players, there exists a unique Nash equilibrium.

Proof. The strict concavity of $w_{i}$ in $x_{i}$ implies that for any $i$ and fixed $x_{i}, w_{3-i}(\bar{x})$ attains its maximum at a unique point $x_{3-i}$. Thus we may define the mapping:

$$
A: X_{1} \times X_{2} \rightarrow X_{1} \times X_{2}
$$

such that for $\left(x_{1}, x_{2}\right)=A\left(y_{1}, y_{2}\right)$ we have

$$
x_{1}=\underset{x \in X_{1}}{\operatorname{argmax}} w_{1}\left(x, y_{2}\right), \quad x_{2}=\underset{x \in X_{2}}{\operatorname{argmax}} w_{2}\left(y_{1}, x\right) .
$$

Put $A_{i}\left(y_{1}, y_{2}\right):=x_{i}$ for $i=1,2$. We will prove now that

$$
\|A(\bar{x})-A(\bar{y})\|_{\infty}<\|\bar{x}-\bar{y}\|_{\infty}
$$

for $\bar{x}, \bar{y} \in X_{1} \times X_{2}$.

Consider the case in which both $A_{i}(\bar{x})$ and $A_{i}(\bar{y})$ are inside the interval $X_{i}$. Then

$$
\frac{\partial w_{1}}{\partial x_{1}}\left(A_{1}(\bar{x}), x_{2}\right)=\frac{\partial w_{1}}{\partial x_{1}}\left(A_{1}(\bar{y}), y_{2}\right)=0
$$

and Lemma 5.1 implies

$$
\left|A_{1}(\bar{x})-A_{1}(\bar{y})\right|<\left\|\left(A_{1}(\bar{x}), x_{2}\right)-\left(A_{1}(\bar{y}), y_{2}\right)\right\|_{\infty}=\left|x_{2}-y_{2}\right| .
$$

A similar inequality can be obtained for $A_{2}$ :

$$
\left|A_{2}(\bar{x})-A_{2}(\bar{y})\right|<\left\|\left(x_{1}, A_{2}(\bar{x})\right)-\left(y_{1}, A_{2}(\bar{y})\right)\right\|_{\infty}=\left|x_{1}-y_{1}\right| .
$$

From (15) and (16) it can be easily concluded that (14) holds.

If any of the values $A_{i}(\bar{x})$ or $A_{i}(\bar{y})$ belongs to the edge of the interval $X_{i}$, we may define (thanks to the fact that the derivatives $w_{i}$ are finite and $X_{i}$ are closed) the function $\tilde{w}_{i}: R \times R \rightarrow R$ such that $\tilde{w}_{i}(\bar{x})=w_{i}(\bar{x})$ for $\bar{x} \in X_{1} \times X_{2}$ with the same properties like $w_{i}$, that is strict concavity in $x_{i}$ and Assumption 5.1.

For these functions we conduct similar reasoning, as in the case of $A_{i}(\bar{x})$ and $A_{i}(\bar{y})$ inside the set $X_{1} \times X_{2}$.

Define $\tilde{A}$ for the function $\tilde{w}_{i}$ just as $A$ was defined for $w_{i}$. Note that

$$
A_{i}(\bar{x}) \in\left[\tilde{A}_{i}(\bar{x}), \tilde{A}_{i}(\bar{y})\right] .
$$

This implies that

$$
\left|A_{i}(\bar{x})-A_{i}(\bar{y})\right| \leq\left|\tilde{A}_{i}(\bar{x})-\tilde{A}_{i}(\bar{y})\right|<\|\bar{x}-\bar{y}\|_{\infty},
$$

and this ends the proof of (14).

Note that there exists a Nash equilibrium in the considered game (see Remark 5.1). Assume that there exist two different Nash equilibria: $\bar{x}^{*} \in X_{1} \times X_{2}$ and $\bar{y}^{*} \in X_{1} \times X_{2}$. Note that these are fixed points of the mapping $A$, i.e. $\bar{x}^{*}=A\left(\bar{x}^{*}\right)$ and $\bar{y}^{*}=A\left(\bar{y}^{*}\right)$. Thus

$$
\left\|\bar{x}^{*}-\bar{y}^{*}\right\|_{\infty}=\left\|A\left(\bar{x}^{*}\right)-A\left(\bar{y}^{*}\right)\right\|_{\infty}<\left\|\bar{x}^{*}-\bar{y}^{*}\right\|_{\infty}
$$

and so we have obtained a contradiction. Therefore the game has a unique Nash equilibrium. 
Lemma 5.3. For a fixed $s \in S \subseteq(0, \infty)$ consider a two-person game with the payoff of player $i$ given by

$$
u_{i}\left(x_{i}\right)+k_{i} l\left(s-x_{1}-x_{2}\right),
$$

where $x_{i} \in\left[0, a_{i}(s)\right], a_{1}(s)+a_{2}(s)<s$ and

(i) $u_{i}:[0, \infty) \rightarrow[0, \infty)$ fulfils $u_{i}^{\prime \prime}(x)<0$ for all $x \in(0,+\infty)$ and it is increasing and twice differentiable,

(ii) $k_{i} \in R$,

(iii) $l:[0, \infty) \rightarrow[0, \infty)$ is concave, increasing and twice differentiable.

This game has a unique Nash equilibrium.

Proof. (a) Consider the case that $k_{1} \leq 0$ or $k_{2} \leq 0$. Assume that $k_{2} \leq 0$ (for $k_{1} \leq 0$ the proof is similar). Note that the payoff of player 2: $u_{2}\left(x_{2}\right)+k_{2} l\left(s-x_{1}-x_{2}\right)$ is increasing in $x_{2}$. Thus, the optimal choice of player 2 (independent of the choice of player 1 ) is $x_{2}^{*}=a_{2}(s)$.

If $k_{1}>0$, then the payoff of player $1, u_{1}\left(x_{1}\right)+k_{1} l\left(s-x_{1}-x_{2}\right)$, is strictly concave in $x_{1}$, thus there exists a unique point

$$
x_{1}^{*}=\underset{x_{1} \in\left[0, a_{1}(s)\right]}{\operatorname{argmax}}\left[u_{1}\left(x_{1}\right)+k_{1} l\left(s-x_{1}-x_{2}^{*}\right)\right] .
$$

$\left(x_{1}^{*}, x_{2}^{*}\right)$ is then a unique Nash equilibrium.

(b) If $k_{i} \leq 0$ for $i=1,2$ then a unique Nash equilibrium is $\left(x_{1}^{*}, x_{2}^{*}\right)$, where $x_{1}^{*}=a_{1}(s)$, $x_{2}^{*}=a_{2}(s)$.

(c) Let us consider the case that $k_{i}>0, i=1,2$.

Note that the payoff of player $i$ is strictly concave in $x_{i}$ and both payoffs fulfil Assumption 5.1. Thus the uniqueness of the Nash equilibrium is implied by Lemma 5.2.

\section{References}

[1] R. Amir, Continuous stochastic games of capital accumulation with convex transitions, Games and Econ. Behavior 15 (1996), 111-131.

[2] R. Amir, Stochastic games in economics: the lattice theoretic approach, in: Stochastic Games and Their Applications, A. Neyman and S. Sorin (eds.), NATO Science Series C, Mathematical and Physical Sciences 507, Kluwer, Dordrecht, 2003,

[3] Ł. Balbus and A. S. Nowak, Construction of Nash equilibria in symmetric stochastic games of capital accumulation, Mathematical Methods of Operations Research 60 (2004), 267-277.

[4] D. P. Bertsekas and S. E. Shreve, Stochastic Optimal Control: The Discrete-Time Case, Academic Press, New York, 1978.

[5] D. Blackwell, Discounted dynamic programming, Ann. Math. Statist. 36 (1965), 226-235.

[6] J. Cave, The cold fish war: long-term competition in dynamic game, Rand Journal of Economics 18 (1987).

[7] L. O. Curtat, Markov equilibria of stochastic games with complementarities, Games and Econ. Behavior 17 (1996), 177-199.

[8] N. Dunford and J. Schwartz, Linear Operators, Vol. 1, Wiley, New York, 1957. 
[9] P. Dutta and R. Sundaram, Markovian equilibrium in class of stochastic games: Existence theorems for discounted and undiscounted models, Economic Theory 2 (1992), 197-214.

[10] E. B. Dynkin and A. A. Yushkevich, Controlled Markov Processes and Their Applications, Springer Verlag, 1979.

[11] R. Fisher and L. Mirman, The compleat fish wars: Biological and dynamic interactions, Journal of Environmental Economics and Management 30 (1996), 34-42.

[12] D. Gabay and H. Moulin, On the uniqueness and stability of Nash-equilibria in noncooperative games, in: Applied Stochastic Control in Econometrics and Management Science, A. Bensoussan et al. (eds.), North-Holland, 1980, 271-293.

[13] O. Hernandez-Lerma, Adaptive Markov Control Processes, Springer-Verlag, New York, 1989.

[14] D. Levhari and L. Mirman, The great fish war: An example using a dynamic Cournot-Nash solution, Bell J. Econ. 11 (1980), 322-344.

[15] M. K. Majumdar and R. K. Sundaram, Symmetric stochastic games of resource extraction: The existence of non-randomized stationary equilibrium, in: Stochastic Games and Related Topics, T. Ferguson et al. (eds.), Kluwer Academic Publishing, 1988.

[16] R. Mendelsohn and M. Sobel, Capital accumulation and the optimization of renewable resources, J. Econ. Theory 23 (1981), 243-260.

[17] J. J. Moré, Coercivity conditions in non-linear complementary problems, SIAM Review 16 (1974), 1-16.

[18] J. F. Nash, Equilibrium points in n-person games, Proc. Nat. Acad. Sci. U.S.A. 36 (1950), $48-49$.

[19] J. Neveu, Mathematical Foundations of the Calculus of Probability, Holden-Day, San Francisco, 1965.

[20] A. S. Nowak, On a new class of nonzero-sum discounted stochastic games having stationary Nash equilibrium points, Internat. J. Game Theory 32 (2003), 121-132.

[21] A. S. Nowak, N-person stochastic games: extensions of the finite state space case and correlation, in: Stochastic Games and Their Applications, A. Neyman and S. Sorin (eds.), NATO Science Series C, Mathematical and Physical Sciences, 507, Kluwer Academic Publishers, Dordrecht, 2003, 93-106.

[22] A. S. Nowak and P. Szajowski, On Nash equilibria in stochastic games of capital accumulation, Game Theory and Appl. 9 (2003), 118-129.

[23] D. Smart, Fixed-Point Theorems, Cambridge University Press, 1974.

[24] M. Sobel, Stochastic fishery game with myopic equilibria, in: Essays in the Economics of Renewable Resources, L. Mirman, D. Spulber (eds.), North-Holland, 1982, 259-268.

[25] R. K. Sundaram, Perfect equilibrium in a class of symmetric dynamic games, J. Econ. Theory 47 (1989), 153-177.

[26] P. Szajowski, Constructions of Nash equilibria in stochastic games of resource extraction with additive transition atructure, Math. Methods of Operations Research (ZOR) 2006, $1-22$.

[27] D. Topkis, Minimizing a submodular function on a lattice, Operations Research 26 (1978), 305-321.

[28] P. Więcek, Continuous convex stochastic games of capital accumulation, Ann. of Dynamic Games, 2003. 